\title{
Lie Tori-A Simple Characterization of Extended Affine Lie Algebras
}

\author{
By
}

Yoji Yoshï*

\begin{abstract}
We show the existence of a nonzero graded form on a Lie torus by the existence of a nonzero graded form on a structurable torus. This gives a simple characterization of the core of an extended affine Lie algebra (EALA). Namely, the core of any EALA is a Lie torus, and any centreless Lie torus is the centreless core of some EALA. We also show that a graded form on a Lie torus is unique up to scalars.
\end{abstract}

\section{Introduction}

The core of an extended affine Lie algebra (or an EALA for short) is itself already a very interesting object. It is a natural but not too wide generalization of the derived algebra of an affine Kac-Moody Lie algebra. (The derived algebra is often called an affine Kac-Moody Lie algebra too.) Our motivation in this paper is to find a simple and direct definition of the core that does not depend intrinsically on an EALA. Also, the classification of the cores seems not depending on specific properties of the base field, even though EALAs are only defined over $\mathbb{C}$ (the field of complex numbers). Moreover, the classification suggests a strong connection between EALAs and finite-dimensional simple isotropic Lie algebras, and therefore the base field should be allowed to be non-algebraically closed.

Thus we have picked three rather simple axioms to define a Lie algebra over a field $F$ of characteristic 0 corresponding to the core of an EALA. We call such a Lie algebra a Lie $n$-torus or simply a Lie torus. It turns out that

Communicated by K. Saito. Received May 11, 2004.

2000 Mathematics Subject Classification(s): 17B67, 17B70, 17 D99.

*Department of Mathematics, North Dakota State University, Fargo, ND, 58105-5075 USA.

e-mail: yoji.yoshii@ndsu.edu.

(C) 2006 Research Institute for Mathematical Sciences, Kyoto University. All rights reserved. 
these choices work well, and a Lie $n$-torus over $\mathbb{C}$ characterizes the core of an EALA with nullity $n$.

We now give a rough explanation of the three axioms. (The precise definition is in Definition 1.3.) First, we require that the Lie algebra is graded by both a finite irreducible root system $\Delta$ (possibly nonreduced) and the abelian group $\mathbb{Z}^{n}$. Affine Kac-Moody Lie algebras have such a double grading for $n=1$. Second, we pick one of the most important and basic properties of finite-dimensional simple isotropic Lie algebras, which we call the division property. Roughly speaking, this property is equivalent to the invertibility in the (unital) coordinate algebra attached to the Lie algebra. The Lie algebras satisfying these 2 axioms, called division $\left(\Delta, \mathbb{Z}^{n}\right)$-graded Lie algebras, are already interesting, but their classification seems difficult. Thus we assume, as the third axiom, that the dimensions of certain homogeneous spaces relative to the double grading above are equal to or less than 1 (called 1-dimensionality). By adding this assumption, the coordinate algebra becomes a very concrete object which is a natural analogue of the algebra of Laurent polynomials in $n$-variables in several classes of unital algebras (associative, alternative, Jordan or structurable algebras). We call it an $n$-torus or simply a torus. If the torus is associative, it is a twisted group algebra $F^{t}\left[\mathbb{Z}^{n}\right]$, or in other words, a quantum torus $F_{q}\left[t_{1}^{ \pm 1}, \ldots, t_{n}^{ \pm 1}\right]$. In some sense tori are the most tractable infinite-dimensional algebras, and they are concrete (more tractable and concrete than finite-dimensional division algebras though a torus is never a division algebra). Also, most tori can be embedded into certain finite-dimensional division algebras over certain fields.

Besides their connection with the theory of EALAs, Lie tori may be relevant to the study of $\mathbb{Z}^{n}$-graded Lie algebras. A Lie $n$-torus modulo its centre, called a centreless Lie $n$-torus, is a $\mathbb{Z}^{n}$-graded Lie algebra which is graded simple (see [Y5, Lem.4.4]). Such algebras for $n=1$, i.e., $\mathbb{Z}$-graded Lie algebras which are graded simple were classified by Mathieu $[\mathrm{M}]$ with certain condition on the dimensions of homogeneous spaces. The classification of $\mathbb{Z}^{n}$-graded Lie algebras which are graded simple is a big open problem (see [OZ] for some results on the $\mathbb{Z}^{2}$-graded Lie algebras with certain restrictions). Toward the classification of such graded Lie algebras, centreless Lie $n$-tori would serve as counterpart of (possibly twisted) loop algebras in the case of $n=1$.

It is easy to see that the core of an EALA is a Lie torus. The problem is the converse. We need to find a nonzero graded form on a Lie torus, because one of the EALA axioms requires the existence of a nondegenerate "form". (We simply use the term form for an invariant symmetric bilinear form.) Since 
Lie tori are infinite-dimensional, the existence of such a form was not so easy to show. For the preparation, we first prove in Lemma 2.1 that a nonzero graded form on a Lie torus has very strong properties. For example, the induced form on the centreless Lie torus is nondegenerate. From these properties we know how such a form should be defined. Also, by this lemma, we prove that a graded form on a Lie torus is unique up to scalars (Theorem 2.2).

Then we prove for a $\Delta$-graded Lie algebra $L=\oplus_{\mu \in \Delta \cup\{0\}} L_{\mu}$ that the existence of a certain bilinear form on the space $\oplus_{\mu \in \Delta} L_{\mu}$ guarantees the existence of a form on $L$ (Lemma 4.1). This is a similar trick which was used for showing the existence of a form on the Tits-Kantor-Koecher Lie algebra associated to a Jordan algebra if the Jordan algebra has a form, or more generally, the existence of a form on the Kantor Lie algebra associated to a structurable algebra if the structurable algebra has a form (Schafer $[\mathrm{S}]$ ). It is also similar to the method of showing that a symmetrizable Kac-Moody Lie algebra has a form (e.g. $[\mathrm{K}, \mathrm{p} .18])$. By the trick, we can reduce the problem to the case of Lie tori of type $\mathrm{BC}_{1}$.

A Lie torus of type $\mathrm{BC}_{1}$ is coordinatized by a structurable torus (see $\S 6$ and Lemma 6.1). We will show that the existence of a nonzero graded form on a structurable torus guarantees the existence of a nonzero graded form on a Lie torus of type $\mathrm{BC}_{1}$ (Corollary 3.6), using Schafer's result mentioned above (see Lemma 6.3 and Remark 6.4). Thus our problem now is to show the existence of a nonzero graded form on a structurable torus.

Structurable $n$-tori are not classified yet. However, Allison and the author have recently classified structurable 2 -tori in $[\mathrm{AY}]$. Also, we already knew that a Jordan torus, which is in a subclass of structurable tori, admits a nondegenerate graded form (see $[\mathrm{NY}]$ ). Using these results, we can show that any structurable $n$-torus admits a nondegenerate graded form (Theorem 5.2). Thus we solve the problem, that is, any centreless Lie torus over $\mathbb{C}$ is the centreless core of some EALA.

In the final section, we construct an EALA over $F$ from a centreless Lie torus over $F$ (not necessarily over $\mathbb{C}$ ), by the method used in [AABGP, Ch.III]. One finds that such an EALA also has an extended affine root system, using the existence of the form above. Also, we construct a certain EALA similar to so-called degenerate EALAs studied in [G].

We do not dispute the relationship between Lie tori and elliptic Lie algebras given in $[\mathrm{SY}]$. However, one can see from [SY] and [Ya] that Lie 2-tori are homomorphic images of elliptic Lie algebras. It would be interesting to consider whether this holds in greater generality. 
The organization of the paper is as follows. In $\S 1$ we define a Lie torus and a graded form. In $\S 2$ we prove some properties and uniqueness of the graded forms explained above. In $\S 3$ we give a key lemma for the existence of a form on a $\Delta$-graded Lie algebra. In $\S 4$ we review a characterization of the centreless core of an EALA in [AABGP, Ch.III], and explain our purpose and goal in connection with Lie tori. In $\S 5$ we define a structurable torus. Then the existence of a nondegenerate graded form on a structurable torus is shown. In $\S 6$ we review Kantor Lie algebras, and state the coordinatization theorem of Lie tori of type $\mathrm{BC}_{1}$. Then we show that a Lie torus of type $\mathrm{BC}_{1}$ admits a nonzero graded form. In $\S 7$ we finally show that a Lie torus of any type admits a nonzero graded form. In the last section we discuss EALAs over $F$.

Finally, I would like to thank Professors Bruce Allison, Jun Morita, Erhard Neher, and Maliheh Yousofzadeh for some suggestions and corrections.

\section{$\S 1 . \quad$ Basic Concepts}

Throughout the paper the base field $F$ has characteristic 0 . Let $\Delta$ be a finite irreducible root system, i.e., $\Delta=\mathrm{A}_{l}(l \geq 1), \mathrm{B}_{l}\left(l \geq 1, \mathrm{~B}_{1}=\mathrm{A}_{1}\right), \mathrm{C}_{l}$ $\left(l \geq 2, \mathrm{C}_{2}=\mathrm{B}_{2}\right), \mathrm{D}_{l}(l \geq 4), \mathrm{E}_{l}(l=6,7,8), \mathrm{F}_{4}, \mathrm{G}_{2}$ and $\mathrm{BC}_{l}(l \geq 1)$. Let

$$
\Delta^{\text {red }}:= \begin{cases}\Delta & \text { if } \Delta \text { is reduced } \\ \left\{\mu \in \Delta \mid \mu \text { is reduced, i.e., } \frac{1}{2} \mu \notin \Delta\right\} & \text { otherwise, i.e., if } \Delta=\mathrm{BC}_{l} .\end{cases}
$$

Note that $\Delta^{\text {red }}=\mathrm{B}_{l}$ if $\Delta=\mathrm{BC}_{l}$. Let $\mathfrak{g}$ be a finite-dimensional split simple Lie algebra over $F$ with a split Cartan subalgebra $\mathfrak{h}$ and the root system $\Delta^{\text {red }}$ so that $\mathfrak{g}$ has the root space decomposition $\mathfrak{g}=\mathfrak{h} \oplus\left(\oplus_{\mu \in \Delta^{\text {red }}} \mathfrak{g}_{\mu}\right)$ with $\mathfrak{h}=\mathfrak{g}_{0}$. A $\Delta$-graded Lie algebra $L$ over $F$ with grading subalgebra $\mathfrak{g}$ or grading pair $(\mathfrak{g}, \mathfrak{h})$ is defined as

(i) $L$ contains $\mathfrak{g}$ as a subalgebra;

(ii) $L=\oplus_{\mu \in \Delta \cup\{0\}} L_{\mu}$, where $L_{\mu}=\{x \in L \mid[h, x]=\mu(h) x$ for all $h \in \mathfrak{h}\}$; and

(iii) $L_{0}=\sum_{\mu \in \Delta}\left[L_{\mu}, L_{-\mu}\right]$.

Note that the Jacobi identity implies $\left[L_{\mu}, L_{\nu}\right] \subset L_{\mu+\nu}$ for all $\mu, \nu \in \Delta \cup\{0\}$. $\left(L_{\mu+\nu}:=0\right.$ if $\mu+\nu \notin \Delta \cup\{0\}$.) Also, the centre $Z=Z(L)$ of $L$ is contained in $L_{0}$, and $L / Z$ is again a $\Delta$-graded Lie algebra with $Z(L / Z)=0$. A $\Delta$-graded Lie algebra having trivial centre is called centreless.

Remark 1.1. (i) The $\Delta$-graded Lie algebras for a reduced root system $\Delta$ were introduced by Berman and Moody $[\mathrm{BM}]$, and have been recently generalized for a nonreduced case by Allison, Benkart and Gao [ABG1]. They 
classified more general $\mathrm{BC}_{l}$-graded Lie algebras in [ABG1] (and [BS] for $l=1$ ) than our concept above, and so we only consider a special class of $\mathrm{BC}_{l}$-Lie algebras in their sense. The reason comes from the theory of extended affine Lie algebras (EALAs). Namely, the core of an EALA is a $\Delta$-graded Lie algebra in our sense, which was shown in [AG, Proposition 1.16].

(ii) $\mathrm{A}_{l}$-graded Lie algebra is considered as a $\mathrm{BC}_{l}$-graded Lie algebra. In particular, an $\mathrm{A}_{1}$-graded Lie algebra is considered as a $\mathrm{BC}_{1}$-graded Lie algebra.

Definition 1.2. We call a symmetric invariant bilinear form on a Lie algebra $L$ simply a form. Here 'invariant' is in the sense that $([x, y], z)=(x,[y, z])$ for all $x, y, z \in L$. Note that if a $\Delta$-graded Lie algebra $L=\oplus_{\mu \in \Delta \cup\{0\}} L_{\mu}$ has a form $(\cdot, \cdot)$, then $\left(L_{\mu}, L_{\nu}\right)=0$ unless $\mu+\nu=0$ for $\mu, \nu \in \Delta \cup\{0\}$.

Throughout the paper let $G=(G,+, 0)$ be an abelian group. We will consider a $G$-graded Lie algebra $L=\oplus_{g \in G} L^{g}$, which is a $G$-graded vector space satisfying $\left[L^{g}, L^{h}\right] \subset L^{g+h}$ for all $g, h \in G$. For convenience, we always assume that

$$
\operatorname{supp} L:=\left\{g \in G \mid L^{g} \neq 0\right\} \text { generates } G \text {. }
$$

\section{Definition 1.3.}

(1) A form $(\cdot, \cdot)$ on a $G$-graded Lie algebra $L=\oplus_{g \in G} L^{g}$ satisfying

$$
\left(L^{g}, L^{h}\right)=0 \quad \text { unless } g+h=0 \text { for all } g, h \in G,
$$

is called a graded form.

(2) A $\Delta$-graded Lie algebra $L=\oplus_{\mu \in \Delta \cup\{0\}} L_{\mu}$ with grading subalgebra $\mathfrak{g}$ is called $(\Delta, G)$-graded if $L=\oplus_{g \in G} L^{g}$ is a $G$-graded Lie algebra such that $\mathfrak{g} \subset L^{0}$. Then we have

$$
L=\bigoplus_{\mu \in \Delta \cup\{0\}} \bigoplus_{g \in G} L_{\mu}^{g},
$$

where $L_{\mu}^{g}=L_{\mu} \cap L^{g}$ since $L^{g}$ is an $\mathfrak{h}$-submodule of $L$.

(3) Let $Z(L)$ be the centre of $L$ and let $\mu^{\vee} \in \mathfrak{h}$ for $\mu \in \Delta$ be the coroot of $\mu$. Then $L$ is called a division $(\Delta, G)$-graded Lie algebra if for any $\mu \in \Delta$ and any $0 \neq x \in L_{\mu}^{g}$, there exists $y \in L_{-\mu}^{-g}$ such that $[x, y] \equiv \mu^{\vee}$ modulo $Z(L)$. (division property) 
(4) A division $(\Delta, G)$-graded Lie algebra $L=\oplus_{\mu \in \Delta \cup\{0\}} \oplus_{g \in G} L_{\mu}^{g}$ is called a Lie $G$-torus of type $\Delta$ if

$$
\operatorname{dim}_{F} L_{\mu}^{g} \leq 1 \text { for all } g \in G \text { and } \mu \in \Delta \text {. (1-dimensionality) }
$$

If $G=\mathbb{Z}^{n}$, we call it a Lie $n$-torus or simply a Lie torus.

The following two lemmas are easy consequence from the definition.

Lemma 1.4. Let $L=\oplus_{\mu \in \Delta \cup\{0\}} \oplus_{g \in G} L_{\mu}^{g}$ be a Lie G-torus with grading pair $(\mathfrak{g}, \mathfrak{h})$ and its centre $Z$. Then $L / Z$ is a Lie $G$-torus with the same grading pair $(\mathfrak{g}, \mathfrak{h})$. (We identify any subspace of $L$ and its natural image on $L / Z$ if it maps injectively.) Also, $\mathfrak{g}_{\mu}=L_{\mu}^{0}$ for all $\mu \in \Delta^{\text {red }}$ and $\mathfrak{h} \oplus Z^{0}=L_{0}^{0}$, where $Z^{0}=Z \cap L^{0}$.

Proof. Since $Z$ is $G$-graded and contained in $L_{0}$, the first assertion is clear. By 1-dimensionality, $\mathfrak{g}_{\mu}=L_{\mu}^{0}$ for all $\mu \in \Delta^{\text {red }}$. By the division property and 1-dimensionality, $\left[L_{\mu}^{g}, L_{-\mu}^{-g}\right] \equiv F \mu^{\vee} \bmod Z^{0}$ for all $\mu \in \Delta$ and $g \in G$, and so $\mathfrak{h} \oplus Z^{0}=L_{0}^{0}$.

Lemma 1.5. Let $L$ be a $\Delta$-graded Lie algebra with its centre $Z$ and $a$ form $(\cdot, \cdot)$. Then $Z \subset \operatorname{rad}(\cdot, \cdot)$, where $\operatorname{rad}(\cdot, \cdot):=\{x \in L \mid(x, L)=0\}$ is the radical of $(\cdot, \cdot)$. Also, if $L / Z$ has a form $(\cdot, \cdot)$, then $L$ has a unique extended form $(\cdot, \cdot)^{r}$ such that $(x, y)^{-}=(x+Z, y+Z)$ for all $x, y \in L$.

Proof. Since $Z \subset L_{0}$, we have $\left(Z, L_{\mu}\right)=0$ for $\mu \neq 0$. Since $L_{0}=$ $\sum_{\mu \in \Delta}\left[L_{\mu}, L_{-\mu}\right]$, we get $(Z, L)=0$. The second statement is clear.

\section{$\S 2$. Some Properties of Graded Forms}

Our main object in the paper is a Lie torus, but we consider a Lie $G$-torus since the abelian group $G$ need not be $\mathbb{Z}^{n}$ in this section.

Lemma 2.1. Let $L=\oplus_{\mu \in \Delta \cup\{0\}} \oplus_{g \in G} L_{\mu}^{g}$ be a division G-graded Lie algebra with grading pair $(\mathfrak{g}, \mathfrak{h})$ and its centre $Z$. Suppose that $L$ admits a nonzero graded form $(\cdot, \cdot)$. Then:

$$
Z=\operatorname{rad}(\cdot, \cdot)
$$

If $L$ is a Lie G-torus, then

$$
\left.(\cdot, \cdot)\right|_{\mathfrak{h} \times \mathfrak{h}} \text { is nondegenerate, and }
$$




$$
\left[x_{\mu}^{g}, x_{-\mu}^{-g}\right] \equiv\left(x_{\mu}^{g}, x_{-\mu}^{-g}\right) t_{\mu} \quad \bmod Z
$$

for any $x_{\mu}^{g} \in L_{\mu}^{g}$ and $x_{-\mu}^{-g} \in L_{-\mu}^{-g}$, where $t_{\mu}$ for $\mu \in \Delta \cup\{0\}$ is a unique element in $\mathfrak{h}$ such that $\mu(h)=\left(t_{\mu}, h\right)$ for all $h \in \mathfrak{h}$.

Proof. (a): By Lemma 1.5, we only need to show $\operatorname{rad}(\cdot, \cdot) \subset Z$. But this follows from the fact that $L / Z$ is graded simple (see [Y5, Lem.4.4]) since $\operatorname{rad}(\cdot, \cdot)$ is a graded ideal.

(b): Suppose that $\left.(\cdot, \cdot)\right|_{\mathfrak{h} \times \mathfrak{h}}$ is degenerate. So let $0 \neq\left. h \in \operatorname{rad}(\cdot, \cdot)\right|_{\mathfrak{h} \times \mathfrak{h}}$. Since the simple Lie algebra $\mathfrak{g}=(\mathfrak{g}, \mathfrak{h})$ is a subalgebra, there exist $\mu \in \Delta$ and $0 \neq x_{\mu} \in \mathfrak{g}_{\mu}=L_{\mu}^{0}$ such that $\left[h, x_{\mu}\right] \neq 0$. So $\left[h, x_{\mu}\right]=a x_{\mu}$ for some $0 \neq a \in F$. Then there exists $y_{-\mu} \in \mathfrak{g}_{-\mu}=L_{-\mu}^{-0}$ such that $\left[x_{\mu}, y_{-\mu}\right]=\mu^{\vee}$. Hence, $0=\left(h, \mu^{\vee}\right)=\left(h,\left[x_{\mu}, y_{-\mu}\right]\right)=a\left(x_{\mu}, y_{-\mu}\right)$, and we get

$$
\left(x_{\mu}, y_{-\mu}\right)=0 .
$$

Also, we have $0=\left(2 x_{\mu}, y_{-\mu}\right)=\left(\left[\mu^{\vee}, x_{\mu}\right], y_{-\mu}\right)=\left(\mu^{\vee},\left[x_{\mu}, y_{-\mu}\right]\right)=\left(\mu^{\vee}, \mu^{\vee}\right)$. Then, for any $g \in G$ with $0 \neq x_{\mu}^{g} \in L_{\mu}^{g}$, by the division property (and Lemma 1.5), there exists $0 \neq y_{-\mu}^{-g} \in L_{-\mu}^{-g}$ so that $\left(x_{\mu}^{g}, y_{-\mu}^{-g}\right)=\frac{1}{2}\left(\left[\mu^{\vee}, x_{\mu}^{g}\right], y_{-\mu}^{-g}\right)=$ $\frac{1}{2}\left(\mu^{\vee}, \mu^{\vee}\right)=0$. Hence, by 1-dimensionality, $\left(L_{\mu}^{g}, L_{-\mu}^{-g}\right)=0$ for all $g \in G$, and so $\left(L_{\mu}, L_{-\mu}\right)=0$.

Now, for any $\nu \in \Delta$ and $g \in G$ with $0 \neq x_{\nu}^{g} \in L_{\nu}^{g}$, there exists $0 \neq$ $y_{-\nu}^{-g} \in L_{-\nu}^{-g}$ again so that $\nu\left(\mu^{\vee}\right)\left(x_{\nu}^{g}, y_{-\nu}^{-g}\right)=\left(\left[\mu^{\vee}, x_{\nu}^{g}\right], y_{-\nu}^{-g}\right)=\left(\mu^{\vee}, \nu^{\vee}\right)$. But, by $(*)$, we have $\left(\mu^{\vee}, \nu^{\vee}\right)=\left(\left[x_{\mu}, y_{-\mu}\right], \nu^{\vee}\right)=\left(x_{\mu},\left[y_{-\mu}, \nu^{\vee}\right]\right)=\mu\left(\nu^{\vee}\right)\left(x_{\mu}, y_{-\mu}\right)=0$. Thus, for any $\nu \in \Delta$ such that $\nu\left(\mu^{\vee}\right) \neq 0$, we get $\left(x_{\nu}^{g}, y_{-\nu}^{-g}\right)=0$. Hence, by 1-dimensionality, $\left(L_{\nu}^{g}, L_{-\nu}^{-g}\right)=0$ for all $g \in G$, and so $\left(L_{\nu}, L_{-\nu}\right)=0$. Repeating this process, we obtain $\left(L_{\nu}, L_{-\nu}\right)=0$ for all $\nu \in \Delta$ since $\Delta$ is irreducible. Therefore, $(L, L)=0$ since $L_{0}=\sum_{\mu \in \Delta}\left[L_{\mu}, L_{-\mu}\right]$. So this is a contradiction.

(c): By a usual trick, we have

$$
\begin{aligned}
\left(\left[x_{\mu}^{g}, x_{-\mu}^{-g}\right], h\right) & =\left(x_{\mu}^{g},\left[x_{-\mu}^{-g}, h\right]\right)=\mu(h)\left(x_{\mu}^{g}, x_{-\mu}^{-g}\right) \\
& =\left(t_{\mu}, h\right)\left(x_{\mu}^{g}, x_{-\mu}^{-g}\right)=\left(\left(x_{\mu}^{g}, x_{-\mu}^{-g}\right) t_{\mu}, h\right)
\end{aligned}
$$

for any $x_{\mu}^{g} \in L_{\mu}^{g}, x_{-\mu}^{-g} \in L_{-\mu}^{-g}$ and all $h \in \mathfrak{h}$. Hence (c) follows from (b) and Lemma $1.4\left(\mathfrak{h} \oplus Z^{0}=L_{0}^{0}\right)$.

Using the lemma, we can show the following.

Theorem 2.2. $\quad$ A graded form on a Lie G-torus is unique up to scalars. 
Proof. Suppose that $(\cdot, \cdot)$ and $(\cdot, \cdot)^{\prime}$ are nonzero graded forms on a Lie $G$-torus $L$. Let $L=\oplus_{\mu \in \Delta \cup\{0\}} \oplus_{g \in G} L_{\mu}^{g}$ with grading pair $(\mathfrak{g}, \mathfrak{h})$ and its centre $Z$. Then, by Lemma 2.1, we have in $L / Z$

$$
\left(x_{\mu}^{g}, x_{-\mu}^{-g}\right) t_{\mu}=\left(x_{\mu}^{g}, x_{-\mu}^{-g}\right)^{\prime} t_{\mu}^{\prime}
$$

for any $x_{\mu}^{g} \in L_{\mu}^{g}$ and $x_{-\mu}^{-g} \in L_{-\mu}^{-g}$, where $t_{\mu}$ and $t_{\mu}^{\prime}$ for $\mu \in \mathfrak{h}^{*}$ are unique elements in $\mathfrak{h}$ such that $\left(t_{\mu}, h\right)=\mu(h)=\left(t_{\mu}^{\prime}, h\right)^{\prime}$ for all $h \in \mathfrak{h}$. Also, $\left.(\cdot, \cdot)\right|_{\mathfrak{h} \times \mathfrak{h}}$ and $\left.(\cdot, \cdot)^{\prime}\right|_{\mathfrak{h} \times \mathfrak{h}}$ are both nonzero, and so $\left.(\cdot, \cdot)\right|_{\mathfrak{g} \times \mathfrak{g}}$ and $\left.(\cdot, \cdot)^{\prime}\right|_{\mathfrak{g} \times \mathfrak{g}}$ are both nondegenerate. Thus there exists $0 \neq c \in F$ such that $\left.(\cdot, \cdot)^{\prime}\right|_{\mathfrak{g} \times \mathfrak{g}}=\left.c(\cdot, \cdot)\right|_{\mathfrak{g} \times \mathfrak{g}}$. Since $\left(t_{\mu}, h\right)=\mu(h)=c\left(t_{\mu}^{\prime}, h\right)$ for all $h \in \mathfrak{h}$, we get $t_{\mu}=c t_{\mu}^{\prime}$. Hence, $c\left(x_{\mu}^{g}, x_{-\mu}^{-g}\right)=$ $\left(x_{\mu}^{g}, x_{-\mu}^{-g}\right)^{\prime}$, and so $\left.c(\cdot, \cdot)\right|_{L_{\mu}^{g} \times L_{-\mu}^{-g}}=\left.(\cdot, \cdot)^{\prime}\right|_{L_{\mu}^{g} \times L_{-\mu}^{-g}}$. Since $\left.(\cdot, \cdot)\right|_{L_{\mu}^{g} \times L_{\nu}^{k}}=\left.(\cdot, \cdot)^{\prime}\right|_{L_{\mu}^{g} \times}$ $L_{\nu}^{k}=0$ for $\mu+\nu \neq 0$ or $g+k \neq 0$, we have $\left.c(\cdot, \cdot)\right|_{L^{\prime} \times L^{\prime}}=\left.(\cdot, \cdot)^{\prime}\right|_{L^{\prime} \times L^{\prime}}$, where $L^{\prime}:=\oplus_{\mu \in \Delta} L_{\mu}$. Therefore, $c(\cdot, \cdot)=(\cdot, \cdot)^{\prime}$, since $L_{0}=\sum_{\mu \in \Delta}\left[L_{\mu}, L_{-\mu}\right]$.

Remark 2.3. The theorem is false if the form is not graded. In fact, it is easy to give such an example using a loop algebra (which is a Lie 1-torus). Note that the last sentence of the exercise 2.5 in $[\mathrm{K}]$ should be modified. (The derived algebra of an affine Kac-Moody Lie algebra is a Lie 1-torus.)

\section{§3. The Centreless Core of an EALA}

A typical example of Lie tori is the core of an EALA. In fact, it is shown in $[A G, \S 1]$ except division property. The division property is shown in [Y2, Ex.2.8(b)] or [AY, Lem.5.5]). The main purpose of this paper is to show that any Lie torus over $\mathbb{C}$ is centrally isogeneous to the core of some EALA. For this purpose, we review a characterization of the centreless core (the core modulo its centre) of an EALA in [AABGP, Ch.III] (see also [Az]). Namely, the centreless core of an EALA with nullity $n$ is characterized as a $\mathbb{Z}^{n}$-graded Lie algebra $L=$ $\oplus_{\sigma \in \mathbb{Z}^{n}} L^{\sigma}$ over $\mathbb{C}$ satisfying the following 8 conditions: (We slightly simplified and modified the conditions there for the use of our notation.)

(1) $L$ has a nondegenerate $\mathbb{Z}^{n}$-graded form $(\cdot, \cdot)$.

(2) There exists a nontrivial finite-dimensional abelian subalgebra $\mathfrak{h}$ of $L$ such that $\operatorname{ad}_{L} \mathfrak{h}$ is diagonalizable. Thus $L=\oplus_{\mu \in \mathfrak{h}^{*}} L_{\mu}$, where $\mathfrak{h}^{*}$ is the dual space of $\mathfrak{h}$ and $L_{\mu}=\{x \in L \mid[h, x]=\mu(h) x$ for all $h \in \mathfrak{h}\}$. We put $\Delta=\left\{\mu \in \mathfrak{h}^{*} \mid \mu \neq 0\right.$ and $\left.L_{\mu} \neq 0\right\}$.

(3) $\left.(\cdot, \cdot)\right|_{\mathfrak{h} \times \mathfrak{h}}$ is nondegenerate.

Thus we may transfer $(\cdot, \cdot)$ to a form on $\mathfrak{h}^{*}$ (the dual space of $\mathfrak{h}$ ). 
(4) $L$ is generated by $L_{\mu}$ for $\mu \in \Delta$.

(5) The restriction of the form $(\cdot, \cdot)$ to the real space $V$ spanned by $\Delta$ is a positive definite real valued form such that $\Delta$ is an irreducible finite root system in $V$ relative to the form $(\cdot, \cdot)$.

(6) $L_{\mu}=\oplus_{\sigma \in \mathbb{Z}^{n}} L_{\mu}^{\sigma}$ for all $\mu \in \Delta$, where $L_{\mu}^{\sigma}:=L_{\mu} \cap L^{\sigma}$.

(7) $L_{\mu}^{0} \neq 0$ for all $\mu \in \Delta^{\text {red }}$.

(8) $\mathfrak{h}=L_{0}^{0}$.

It is not so difficult to show directly that a Lie algebra satisfying (1)-(8) is a centreless Lie torus. Thus,

(3.1) a Lie algebra satisfying (1)-(8) above is a centreless Lie torus,

and our purpose is to show the converse.

Lemma 3.2. If a centreless Lie torus over $\mathbb{C}$ admits a nonzero graded form, then it satisfies (1)-(8) above. More generally, if a Lie torus $L$ over $\mathbb{C}$ with its centre $Z$ admits a nonzero graded form, then $L / Z$ satisfies (1)-(8) above.

Proof. (2), (4), (6) and (7) already follow from our definition of a Lie torus. Also, (8) follows from Lemma 1.4. Now, consider first our $\Delta$ of the grading pair $(\mathfrak{g}, \mathfrak{h})$ of $L$ as a root system in the euclidean space spanned by $\Delta$ through the Killing form $\kappa$ on $\mathfrak{g}$ in the standard way (see e.g. [H, p.40]). Next, by Lemma $2.1, L / Z$ has the well-defined nondegenerate graded form $(\cdot, \cdot)$ such that $\left.(\cdot, \cdot)\right|_{\mathfrak{h} \times \mathfrak{h}}$ is nondegenerate, and so $\left.(\cdot, \cdot)\right|_{\mathfrak{g} \times \mathfrak{g}}$ is nondegenerate. Hence $\left.c(\cdot, \cdot)\right|_{\mathfrak{g} \times \mathfrak{g}}=\kappa$ for some $0 \neq c \in \mathbb{C}$. Take the form $c(\cdot, \cdot)$ on $L / Z$ for (1). Then (3) and (5) hold.

To show the existence of a nonzero graded form on any Lie torus, we need to discuss about structurable tori and to show the existence of a nonzero graded form on them.

\section{§4. Standard Trick}

We show a key lemma in this section for the existence of a form on a $\Delta$-graded Lie algebra. This could be called a standard trick since a similar argument was used for symmetrizable Kac-Moody Lie algebras, Tits-KantorKoecher Lie algebras (TKK algebras) or Kantor Lie algebras (see Remark 6.4(ii)). 
Lemma 4.1. (i) Let $L=\oplus_{\mu \in \Delta \cup\{0\}} L_{\mu}$ be a $\Delta$-graded Lie algebra. Suppose $(\cdot, \cdot)$ is a symmetric bilinear form on $L^{\prime}=\oplus_{\mu \in \Delta} L_{\mu}$ as a vector space such that $(\cdot, \cdot)$ is invariant and $\Delta$-graded whenever it is defined, i.e., $([u, v], w)=$ $(u,[v, w])$ if $[u, v], w, u,[v, w] \in L^{\prime}$, and $\left(L_{\mu}, L_{\nu}\right)=0$ unless $\mu+\nu=0$ for $\mu, \nu \in \Delta$. Then $(\cdot, \cdot)$ uniquely extends a form on $L$.

(ii) Let $L=\oplus_{g \in G} L^{g}$ be a $(\Delta, G)$-graded Lie algebra. Suppose $(\cdot, \cdot)$ is a symmetric bilinear form on $L^{\prime}$ (as in (i)) such that $(\cdot, \cdot)$ is invariant and $(\Delta, G)$-graded whenever it is defined, i.e., in addition to the conditions in (i), $\left(L^{\prime} \cap L^{g}, L^{\prime} \cap L^{h}\right)=0$ unless $g+h=0$ for $g, h \in G$. Then $(\cdot, \cdot)$ uniquely extends a graded form on $L$.

Proof. (i): Since $L_{0}=\sum_{\mu \in \Delta}\left[L_{\mu}, L_{-\mu}\right]$, one can use a usual trick. Namely, we define for $x, y \in L_{0}$,

$$
(x, y)=\sum_{\nu \in \Delta}\left(\left[x, y_{\nu}\right], y_{-\nu}\right),
$$

where $y=\sum_{\nu \in \Delta}\left[y_{\nu}, y_{-\nu}\right]$. (Strictly speaking, one should write $y=\sum_{\nu \in \Delta}$ $\sum_{1 \leq i_{\nu} \leq r_{\nu}}\left[y_{\nu}^{i_{\nu}}, y_{-\nu}^{i_{\nu}}\right]$ for $y_{\nu}^{i_{\nu}} \in L_{\nu}$ and $y_{-\nu}^{i_{\nu}} \in L_{-\nu}$, but we suppress this description since the argument below works without any major change.) Then this is well-defined, i.e., independent of the expression for $y$. Indeed, one can use the same trick in $[\mathrm{K}, \mathrm{p} .18]$, but for the convenience of the reader, we include the proof. First, $\left(\left[\left[u_{\mu}, u_{-\mu}\right], v_{\nu}\right], v_{-\nu}\right)=\left(u_{\mu},\left[u_{-\mu},\left[v_{\nu}, v_{-\nu}\right]\right]\right)$ holds for $\mu, \nu \in \Delta$ since

$$
\begin{aligned}
& \begin{aligned}
\left(\left[\left[u_{\mu}, u_{-\mu}\right], v_{\nu}\right], v_{-\nu}\right) & =\left(\left[\left[u_{\mu}, v_{\nu}\right], u_{-\mu}\right], v_{-\nu}\right)+\left(\left[u_{\mu},\left[u_{-\mu}, v_{\nu}\right]\right], v_{-\nu}\right) \\
& =\left(\left[u_{\mu}, v_{\nu}\right],\left[u_{-\mu}, v_{-\nu}\right]\right)+\left(u_{\mu},\left[\left[u_{-\mu}, v_{\nu}\right], v_{-\nu}\right]\right) \\
& =\left(u_{\mu},\left[v_{\nu},\left[u_{-\mu}, v_{-\nu}\right]\right]+\left[\left[u_{-\mu}, v_{\nu}\right], v_{-\nu}\right]\right) \\
& =\left(u_{\mu},\left[u_{-\mu},\left[v_{\nu}, v_{-\nu}\right]\right]\right) \quad \text { if } \mu+\nu \neq 0, \text { and }
\end{aligned} \\
& \begin{aligned}
\left(\left[\left[u_{\mu}, u_{-\mu}\right], v_{-\mu}\right], v_{\mu}\right) \\
\quad=-\left(\left[\left[u_{-\mu}, u_{\mu}\right], v_{-\mu}\right], v_{\mu}\right) \\
=-\left(\left[\left[u_{-\mu}, v_{-\mu}\right], u_{\mu}\right], v_{\mu}\right)-\left(\left[u_{-\mu},\left[u_{\mu}, v_{-\mu}\right]\right], v_{\mu}\right) \\
\quad=\left(\left[u_{\mu},\left[u_{-\mu}, v_{-\mu}\right]\right], v_{\mu}\right)-\left(u_{-\mu},\left[\left[u_{\mu}, v_{-\mu}\right], v_{\mu}\right]\right) \\
\quad=\left(u_{\mu},\left[\left[u_{-\mu}, v_{-\mu}\right], v_{\mu}\right]\right)-\left(\left[\left[u_{\mu}, v_{-\mu}\right], v_{\mu}\right], u_{-\mu}\right) \\
\quad=\left(u_{\mu},\left[\left[u_{-\mu}, v_{-\mu}\right], v_{\mu}\right]\right)-\left(u_{\mu},\left[v_{-\mu},\left[v_{\mu}, u_{-\mu}\right]\right]\right) \quad \text { (by the above) } \\
\quad=\left(u_{\mu},\left[\left[u_{-\mu}, v_{-\mu}\right], v_{\mu}\right]+\left[v_{-\mu},\left[u_{-\mu}, v_{\mu}\right]\right]\right) \\
\quad=\left(u_{\mu},\left[u_{-\mu},\left[v_{-\mu}, v_{\mu}\right]\right]\right) .
\end{aligned}
\end{aligned}
$$


Thus for $x=\sum_{\mu \in \Delta}\left[x_{\mu}, x_{-\mu}\right]$, we have

$$
(x, y)=\sum_{\nu \in \Delta}\left(\left[x, y_{\nu}\right], y_{-\nu}\right)=\sum_{\mu \in \Delta}\left(x_{\mu},\left[x_{-\mu}, y\right]\right)
$$

and hence $(x, y)$ is independent of the expression for $y$, and also $(x, y)$ is given by the second equality independently of the expression for $x$. Also, we have $(x, y)=\sum_{\nu \in \Delta}\left(\left[x, y_{\nu}\right], y_{-\nu}\right)=\sum_{\nu \in \Delta}\left(y_{-\nu},\left[x, y_{\nu}\right]\right)=-\sum_{\nu \in \Delta}\left(y_{-\nu},\left[y_{\nu}, x\right]\right)=$ $-(-y, x)=(y, x)$. We define for $x \in L_{0}$ and $s \in L^{\prime},(x, s)=(s, x)=0$, which is necessary (see the note in Definition 1.2), so that $\left(L_{\mu}, L_{\nu}\right)=0$ unless $\mu+\nu=0$ for all $\mu, \nu \in \Delta \cup\{0\}$. Thus the extended form $(\cdot, \cdot)$ is symmetric, and the uniqueness is clear from our definition. So we only need to show the invariance, and it is enough to show that $\left(\left[u_{\mu}, v_{\nu}\right], w_{-\mu-\nu}\right)=\left(u_{\mu},\left[v_{\nu}, w_{-\mu-\nu}\right]\right)$ for $\mu, \nu \in \Delta \cup\{0\}$. We divide the proof into the four cases, (a) $\mu \neq 0$ and $\nu \neq 0$, (b) $\mu \neq 0$ and $\nu=0$, (c) $\mu=0$ and $\nu \neq 0$, and (d) $\mu=0$ and $\nu=0$. For (a), if $-\mu-\nu \neq 0$, the invariance is one of our assumptions. Otherwise we get $\left(\left[u_{\mu}, v_{-\mu}\right], w_{0}\right)=\left(u_{\mu},\left[v_{-\mu}, w_{0}\right]\right)$ by our definition. (b) is one of our assumptions since $-\mu-\nu \neq 0$. For $(\mathrm{c})$, we get $\left(\left[u_{0}, v_{\nu}\right], w_{-\nu}\right)=\left(u_{0},\left[v_{\nu}, w_{-\nu}\right]\right)$ by our definition. For $(\mathrm{d})$, let $u_{0}=\sum_{\mu \in \Delta}\left[u_{\mu}, u_{-\mu}\right]$. Then

$$
\begin{aligned}
\left(\left[u_{0}, v_{0}\right], w_{0}\right) & =\sum_{\mu \in \Delta}\left(\left[\left[u_{\mu}, u_{-\mu}\right], v_{0}\right], w_{0}\right) \\
& =\sum_{\mu \in \Delta}\left(\left[\left[u_{\mu},, v_{0}\right], u_{-\mu}\right], w_{0}\right)+\sum_{\mu \in \Delta}\left(\left[u_{\mu},\left[u_{-\mu}, v_{0}\right]\right], w_{0}\right) \\
& =\sum_{\mu \in \Delta}\left(u_{\mu},\left[v_{0},\left[u_{-\mu}, w_{0}\right]\right]\right)+\sum_{\mu \in \Delta}\left(u_{\mu},\left[\left[u_{-\mu}, v_{0}\right], w_{0}\right]\right) \\
& =\sum_{\mu \in \Delta}\left(u_{\mu},\left[u_{-\mu},\left[v_{0}, w_{0}\right]\right]\right)=\left(u_{0},\left[v_{0}, w_{0}\right]\right) .
\end{aligned}
$$

(ii): By (i), we only need to show $\left(L_{0}^{g}, L_{0}^{h}\right)=0$ unless $g+h=0$, but this is clear since $\left(x^{g}, y^{h}\right)=\sum_{\nu \in \Delta} \sum_{h=k+l}\left(\left[x^{g}, y_{\nu}^{k}\right], y_{-\nu}^{l}\right)$ for $x^{g} \in L^{g}$ and $y^{h}=\sum_{\nu \in \Delta} \sum_{h=k+l}\left[y_{\nu}^{k}, y_{-\nu}^{l}\right] \in L^{h}$.

\section{$\S 5 . \quad$ Structurable Tori and Their Graded Forms}

We first review structurable algebras and structurable tori. In this section the base field $F$ can be arbitrary but char. $F \neq 2,3$. Let $A=\left(A,{ }^{-}\right)$be a unital algebra with involution - over $F$. For $x, y \in A$, we define $V_{x, y} \in \operatorname{End}_{F} A$ by

$$
V_{x, y}(z):=(x \bar{y}) z+(z \bar{y}) x-(z \bar{x}) y
$$


for $z \in A$. Put $T_{x}=V_{x, 1}$ for $x \in A$. Then $T_{x}(z)=x z+z x-z \bar{x}$. We say $\left(A,^{-}\right)$ is structurable if

$$
\left[T_{z}, V_{x, y}\right]=V_{T_{z} x, y}-V_{x, T_{\bar{z}} y}
$$

for $x, y, z \in A$, where $[\cdot, \cdot]$ is the commutator. Examples of structurable algebras include any alternative algebra with involution and any Jordan algebra with the identity map as involution. For our purpose we give the following example.

Example 5.1. Suppose that $(E,-)$ is an associative algebra with involution. Let $W$ is a left $E$-module with action denoted by $(e, w) \mapsto e \circ w$. Suppose that $\chi: W \times W \longrightarrow E$ is a hermitian form over $\left(E,^{-}\right)$. That is

$$
\chi\left(e \circ w_{1}, w_{2}\right)=e \chi\left(w_{1}, w_{2}\right), \quad \chi\left(w_{1}, e \circ w_{2}\right)=\chi\left(w_{1}, w_{2}\right) \bar{e}
$$

and

$$
\chi\left(w_{1}, w_{2}\right)=\overline{\chi\left(w_{2}, w_{1}\right)}
$$

for $w_{1}, w_{2} \in W$ and $e \in E$. Let $A=E \oplus W$ with product and involution defined respectively by

$\left(e_{1}+w_{1}\right)\left(e_{2}+w_{2}\right)=e_{1} e_{2}+\chi\left(w_{2}, w_{1}\right)+\overline{e_{1}} \circ w_{2}+e_{2} \circ w_{1} \quad$ and $\quad \overline{e+w}=\bar{e}+w$

for $e_{1}, e_{2}, e \in E$ and $w_{1}, w_{2}, w \in W$. The algebra with involution $(A,-)$ is a structurable algebra called the structurable algebra of the hermitian form $\chi$ (see $[\mathrm{A} 1, \S 8])$.

If $u, v \in A$ and $V_{u, v}=$ id, we say that $u$ is (conjugate) invertible in $\left(A,^{-}\right)$ with conjugate inverse $v$ in $(A,-)$. In that case the element $v$ is unique [AH, Lem.6.1(ii)] and denoted by $\hat{u}$. The notion of conjugate invertibility and conjugate inverse coincides with the notion of invertibility and inverse in Jordan algebras when $(A,-)$ is a Jordan algebra with identity involution.

Next we review structurable tori (see $[\mathrm{AY}])$. Let $G=(G,+, 0)$ be an abelian group. A $G$-graded structurable algebra $(A,-)$ is a structurable algebra so that $A=\oplus_{g \in G} A_{g}$ is $G$-graded as a vector space, $\overline{A_{g}} \subset A_{g}$ and $A_{g} A_{k} \subset A_{g+k}$ for all $g, k \in G$.

Let $\left(A,^{-}\right)=A=\oplus_{g \in G} A_{g}$ be a $G$-graded structurable algebra over $F$. We say $(A,-)$ is a structurable $G$-torus if it satisfies the following three conditions:

(1) all nonzero homogeneous elements are invertible;

(2) $\operatorname{dim}_{F} A_{g} \leq 1$ for all $g \in G$;

(3) $\operatorname{supp} A$ generates $G$, 
where $\operatorname{supp} A=\left\{g \in G \mid A_{g} \neq 0\right\}$. In particular, if $G=\mathbb{Z}^{n}$, then $(A,-)$ is called a structurable $n$-torus or simply a structurable torus. We also use the terms, a Jordan $G$-torus, a Jordan $n$-torus and a Jordan torus when $(A,-)$ is a Jordan algebra with identity involution.

In general the invertibility of structurable algebras is not so easy to handle. However, in the class of structurable $G$-tori, it behaves well in the following sense [AY, Prop.3.1]: For $0 \neq x \in A_{g}$, there exists a unique $y \in A_{-g}$ so that $x y=y x=1$ and $\left[L_{x}, L_{y}\right]=\left[R_{x}, R_{y}\right]=0$, where $L$ and $R$ are the left and right multiplication operators. We call the $y$ the inverse of $x$, denoted $x^{-1}$. Moreover, we have $x^{-1}=\overline{\hat{x}}$.

We note that there exists a structurable torus $\left(E \oplus W,^{-}\right)$of a hermitian form so that $E$ is a graded subalgebra and $W$ is a graded $E$-module (see [AY, Ex.4.6]).

Next we need to discuss about forms on a structurable $G$-torus. As in the Lie algebra case we call a symmetric invariant bilinear form $(\cdot, \cdot)$ on a structurable algebra $A=\left(A,{ }^{-}\right)$simply a form. Here 'invariant' means that

$$
(\bar{x}, \bar{y})=(x, y) \quad \text { and } \quad(z x, y)=(x, \bar{z} y)
$$

for all $x, y, z \in A$. A form $(\cdot, \cdot)$ on a structurable $G$-torus $A=\oplus_{g \in G} A_{g}$ satisfying

$$
\left(A_{g}, A_{k}\right)=0 \quad \text { unless } g+k=0 \text { for all } g, k \in G,
$$

is called a graded form. We define an $F$-linear map $\varepsilon$ on $A$ by

$$
\varepsilon= \begin{cases}\text { id } & \text { on } \left.A_{0}=F 1 \text { (identify with } F\right) \\ 0 & \text { on } \oplus_{g \neq 0} A_{g}\end{cases}
$$

and for $x, y \in A$, put

$$
\epsilon(x, y):=\epsilon(\bar{x} y) .
$$

Then $\epsilon(\cdot, \cdot)$ is symmetric and nondegenerate. In fact $\varepsilon(x, y)-\varepsilon(y, x)=\varepsilon(\bar{x} y-$ $\bar{y} x)=0$ since $\bar{x} y-\bar{y} x$ is skew. Also, the radical of $\varepsilon(\cdot, \cdot)$ is homogeneous, and hence must be 0 by the invertibility of a nonzero homogeneous element. But the author does not know whether or not the invariance holds in general. However, we prove the invariance if $G$ is torsion-free. For this purpose we use the following fact from [AY, Thm 9.22]:

Any structurable 2-torus $\left(A,{ }^{-}\right)$can be identified with some structurable 2-torus of a hermitian form, that is, $\left(A,^{-}\right)=\left(E \oplus W,^{-}\right)$(possibly $W=0$ ), or some Jordan 2-torus. 
Theorem 5.2. $\quad$ There exists a nondegenerate graded form on a structurable $G$-torus if $G$ is torsion-free. A graded form of such a torus is unique up to a scalar.

Proof. Let $\left(A,^{-}\right)$be a structurable $G$-torus, say $A=\oplus_{g \in G} A_{g}$. Note that if $x, y, z$ are homogeneous elements of $A$ then $\varepsilon(x, y)-\varepsilon(\bar{x}, \bar{y})=\varepsilon(\bar{x} y-x \bar{y})=$ $\pm \varepsilon(x y-x y)$ and $\varepsilon(z x, y)-\varepsilon(x, \bar{z} y)=\varepsilon((\bar{x} \bar{z}) y-\bar{x}(\bar{z} y))= \pm \varepsilon((x z) y-x(z y))$. Thus, to show that $\varepsilon(\cdot, \cdot)$ is invariant we need to prove that for all homogeneous elements $x, y, z$ we have

$$
[x, y] \in A_{0} \Longrightarrow[x, y]=0
$$

and

$$
(x, y, z) \in A_{0} \Longrightarrow(x, y, z)=0,
$$

where $[x, y]=x y-y x$ is the commutator and $(x, y, z)=(x y) z-x(y z)$ is the associator.

For (1), suppose for contradiction $x$ and $y$ are nonzero homogenous elements of $A$ so that $[x, y] \in A_{0}$ and $[x, y] \neq 0$. Let $x \in A_{g}$, in which case $y \in A_{-g}$. Hence $y=c x^{-1}$ for some $c \in F$. So $[x, y]=c\left[x, x^{-1}\right]=0$. This contradiction proves (1).

For (2), suppose for contradiction that $x, y$ and $z$ are nonzero homogenous elements of $A$ so that $(x, y, z) \in A_{0}$ and $(x, y, z) \neq 0$. Let $x \in A_{g}$ and $y \in A_{k}$, in which case $z \in A_{-g-k}$. Let $A^{\prime}=\left(A^{\prime},{ }^{-}\right)$be the subalgebra of $A$ generated by $x^{ \pm 1}$ and $y^{ \pm 1}$. Then supp $A^{\prime}$ generates a free abelian group of rank 1 or 2 since $G$ is torsion-free. So $A^{\prime}$ is a structurable $n$-torus where $n=1$ or 2 . If $n=1$, we have a contradiction since structurable 1-tori are associative (see [AY, Thm 7.5]). Suppose that $n=2$. If ${ }^{-}$is the identity map, then $A$ is a Jordan torus and it is known using the classification of Jordan tori that $Z(A) \cap(A, A, A)=0$ (see [NY, Prop.4.9]). Consequently in that case we have $A_{0} \cap(A, A, A) \subset Z(A) \cap(A, A, A)=0$, a contradiction. So we can assume that - is not the identity. Then, $\left(A,{ }^{-}\right)$can be identified with the structurable torus $\left(E \oplus W,^{-}\right)$of a hermitian form $\chi[\mathrm{AY}$, Thm 9.22], as mentioned above. Thus $x=e_{1}+w_{1}, y=e_{2}+w_{2}$ and $z=e_{3}+w_{3}$ where $e_{1}, e_{2}, e_{3}$ are homogeneous in $E$ and $w_{1}, w_{2}, w_{3}$ are homogeneous in $W$. Then a direct calculation yields

$$
\begin{aligned}
(x, y, z)= & {\left[\chi\left(w_{2}, w_{1}\right), e_{3}\right]+\left[\chi\left(w_{3}, w_{2}\right), e_{1}\right]+\left[\chi\left(w_{3}, w_{1}\right), \bar{e}_{2}\right] } \\
& +\left[\bar{e}_{2}, \bar{e}_{1}\right] \circ w_{3}+\left[e_{3}, \bar{e}_{1}\right] \circ w_{2}+\left[e_{3}, e_{2}\right] \circ w_{1} \\
& +\chi\left(w_{1}, w_{2}\right) \circ w_{3}-\chi\left(w_{3}, w_{2}\right) \circ w_{1} .
\end{aligned}
$$


Now, by (1), the first three terms on the right hand side of (3) do not yield nonzero elements of $A_{0}$. But neither do the last five terms, since they are in $W$ and $A_{0} \cap W \subset E \cap W=0$. This contradiction proves (2). Hence $\varepsilon(\cdot, \cdot)$ is a nondegenerate graded form.

Finally let $(\cdot, \cdot)$ be any graded form on $(A,-)$. Define a map $g$ from $A$ into $F$ by $g(x)=(1, x)$ for $x \in A$. Then $g=c \varepsilon$ for some $c \in F$. Hence $(x, y)=(1, \bar{x} y)=g(\bar{x} y)=c \varepsilon(\bar{x} y)=c \varepsilon(x, y)$ for $x, y \in A$, and so any graded form is unique up to a scalar.

Remark 5.3. If a structurable $H$-torus has a nonzero graded form, where $H$ is an arbitrary abelian group generated by 2 elements, then one can exclude the condition in Theorem 5.2 that $G$ is torsion-free.

\section{$\S 6 . \quad$ Lie Tori of Type $\mathrm{BC}_{1}$}

We first review Kantor Lie algebras. Let $(A,-)$ be a structurable algebra, and

$$
A^{-}=\{x \in A \mid \bar{x}=-x\} .
$$

The Kantor Lie algebra $K(A,-)$ is constructed as follows. Let

$$
N=\left(A, A^{-}\right), \quad V_{A, A}=\left\{V_{x, y} \mid x, y \in A\right\}
$$

and let $N^{\sim}$ be a copy of $N$. Then $K\left(A,{ }^{-}\right)=N^{\top} \oplus V_{A, A} \oplus N$ has the following Lie bracket

$$
\begin{aligned}
{\left[V_{x, y},(z, s)\right] } & =\left(V_{x, y}(z),(s y) \bar{x}+x(\bar{y} s)\right), \\
{\left[V_{x, y},(z, s)\right] } & =\left(-V_{y, x}(z),-(s x) \bar{y}-y(\bar{x} s)\right)^{\prime}, \\
{[(x, r),(y, s)] } & =(0, x \bar{y}-y \bar{x}), \\
{\left[(x, r)^{r},(y, s)\right] } & =(0, x \bar{y}-y \bar{x})^{r}, \\
{[(x, r),(y, s)] } & =-(s x, 0)^{r}+V_{x, y}+L_{r} L_{s}+(r y, 0),
\end{aligned}
$$

for $x, y, z \in A$ and $s, r \in A^{-}$, where $L$ is the left multiplication operator. We note that $K(A,-)$ is centreless (see $[\mathrm{A}])$. TKK algebras belong to a special class of Kantor Lie algebras. More precisely, if $A^{-}=0$, then $A=\left(A,{ }^{-}\right)$is a Jordan algebra and $K(A)$ is a TKK algebra.

Now, we state the coordinatization theorem of Lie $G$-tori of $\mathrm{BC}_{1}$, which can be proven in the same way as in [AY, Thm 5.6].

Lemma 6.1. Let $L=\oplus_{\mu \in \Delta \cup\{0\}} \oplus_{g \in G} L_{\mu}^{g}$ be a centreless Lie G-torus of type $B C_{1}$. Then $L$ can be identified with the Kantor Lie algebra $K\left(A,{ }^{-}\right)=$ 
$\left(A, A^{-}\right)^{-} \oplus V_{A, A} \oplus\left(A, A^{-}\right)$for some structurable $G$-torus $(A,-)=\oplus_{g \in G} A_{g}$ as follows: Let $\Delta=\{ \pm 2 \lambda, \pm \lambda\}$. Then for all $g \in G$, we have

$$
\begin{gathered}
L_{-2 \lambda}^{g}=\left(0, A_{g}^{-}\right)^{r}, \quad L_{-\lambda}^{g}=\left(A_{g}, 0\right)^{r}, \quad L_{0}^{g}=\sum_{k+l=g} V_{A_{k}, A_{l}}, \\
L_{\lambda}^{g}=\left(A_{g}, 0\right), \quad L_{2 \lambda}^{g}=\left(0, A_{g}^{-}\right) .
\end{gathered}
$$

Remark 6.2. (i) In Lemma 6.1 , if $L$ is of type $\mathrm{A}_{1}$, the structurable $G$ torus is a Jordan $G$-torus (see Remark 1.1(ii)).

(ii) The converse of Lemma 6.1 is also true. More precisely, if $(A,-)$ is a structurable $G$-torus (resp. a Jordan $G$-torus), then $K\left(A,{ }^{-}\right)$is a Lie $G$-torus of type $\mathrm{BC}_{1}$ (resp. type $\mathrm{A}_{1}$ ). In fact, one can define a $G$-grading on $K\left(A,{ }^{-}\right.$) and its grading pair as in Lemma 6.1. Then 1-dimensionality is clear. The division property follows from the fact that $\hat{x} \in A_{-g}$ for $0 \neq x \in A_{g}$ (see $\S 5$ ).

About forms on Kantor Lie algebras, we have the following by Schafer [S, p.115 (25)]:

Lemma 6.3. If a structurable algebra $(A,-)$ has a form $f(\cdot, \cdot)$, then there exists a unique form $u(\cdot, \cdot)$ on $K\left(A,{ }^{-}\right)$such that $u((x, 0),(y, 0))=f(x, y)$ for all $x, y \in A$. In particular, $u\left((0, r),(0, s)^{\dagger}\right)=-\frac{1}{2} f(r, s)$ for all $r, s \in A^{-}$.

Remark 6.4. (i) This lemma was used for showing some property of forms on a finite-dimensional central simple structurable algebra in [S]. However, his proof does not require any condition on a structurable algebra, and so the lemma is true for structurable algebras in general. See also [BS, Thm 9.10 (iii)].

(ii) Benkart [B] showed a theorem similar to Lemma 6.3 for a $\Delta$-graded Lie algebra of a reduced root system $\Delta$ (in terms of its coordinate algebra). The theorem for nonreduced case is in [ABG1] and [BS].

Using this lemma, we show the following:

Lemma 6.5. If any structurable $G$-torus admits a nonzero graded form, then so does any Lie $G$-torus of type $B C_{1}$. Hence, any Lie $G$-torus of type $B C_{1}$ admits a nonzero graded form if $G$ is torsion-free.

Proof. Identify the Lie $G$-torus modulo centre with $K\left(A,{ }^{-}\right)$for some structurable $G$-torus $\left(A,{ }^{-}\right)$by Lemma 6.1 . Let $f(\cdot, \cdot)$ be a nonzero graded form on $\left(A,{ }^{-}\right)$by our assumption. Then the corresponding form $u$ on $K\left(A,{ }^{-}\right)$to $f$ in Lemma 6.3 is nonzero and clearly graded on $K\left(A,{ }^{-}\right)_{ \pm \lambda} \oplus K\left(A,{ }^{-}\right)_{ \pm 2 \lambda}$ since $f$ is graded. Hence $u$ is graded on $K\left(A,{ }^{-}\right)$by the same reason as in the proof 
of Lemma 4.1(ii). Hence, by Lemma 1.5, the Lie $G$-torus admits a nonzero graded form. The second statement follows from Theorem 5.2.

\section{$\S 7$. The Existence of a Nonzero Graded Form on a Lie Toruss}

We are now ready to prove our main theorem.

Theorem 7.1. Any Lie G-torus admits a nonzero graded form if $G$ is torsion-free. In particular, any Lie torus admits a nonzero graded form.

Proof. Let $L=\oplus_{\mu \in \Delta \cup\{0\}} \oplus_{g \in G} L_{\mu}^{g}$ be a Lie $G$-torus with grading pair $(\mathfrak{g}, \mathfrak{h})$ and its centre $Z$. Using the Killing form $\kappa$ on $\mathfrak{g}$, we define $t_{\mu}$ for $\mu \in \mathfrak{h}^{*}$ as a unique element of $\mathfrak{h}$ such that $\mu(h)=\kappa\left(t_{\mu}, h\right)$ for all $h \in \mathfrak{h}$. Then we have $\mu^{\vee}=\frac{2}{\kappa\left(t_{\mu}, t_{\mu}\right)} t_{\mu}$. Since $\left[L_{\mu}^{g}, L_{-\mu}^{-g}\right] \equiv F \mu^{\vee} \bmod Z$ for all $\mu \in \Delta$, one can define a bilinear form $(\cdot, \cdot)$ on $L^{\prime}=\oplus_{\mu \in \Delta} L_{\mu}$ by

$$
\begin{cases}{\left[x_{\mu}^{g}, x_{\nu}^{k}\right] \equiv\left(x_{\mu}^{g}, x_{\nu}^{k}\right) t_{\mu}} & \bmod Z \\ \left(x_{\mu}^{g}, x_{\nu}^{k}\right)=0 & \text { if } \mu+\nu=0 \text { and } g+k=0\end{cases}
$$

for all $\mu, \nu \in \Delta, g, k \in G, x_{\mu}^{g} \in L_{\mu}^{g}$ and $x_{\nu}^{k} \in L_{\nu}^{k}$. for any $x_{\mu}^{g} \in L_{\mu}^{g}$ and $x_{-\mu}^{-g} \in L_{-\mu}^{-g}$,

Since $t_{-\mu}=-t_{\mu},(\cdot, \cdot)$ is symmetric on $L^{\prime}$. To apply Lemma 4.1(ii) we need to show that

$$
([u, v], w)=(u,[v, w]) \text { for } u \in L_{\mu}^{g}, v \in L_{\nu}^{k}, w \in L_{-\mu-\nu}^{-g-k}, \mu \in \Delta \text { and } \mu+\nu \in \Delta .
$$

Suppose first that $\nu \neq q \mu$ for $q \in \mathbb{Q}$. Then $t_{\mu}$ and $t_{\nu}$ are linearly independent. We have

$$
[[u, v], w]=([u, v], w) t_{\mu+\nu}=([u, v], w) t_{\mu}+([u, v], w) t_{\nu}
$$

On the other hand, using the Jacobi identity,

$$
[[u, v], w]=[[u, w], v]+[u,[v, w]]=([u, w], v) t_{-\nu}+(u,[v, w]) t_{\mu} .
$$

Comparing the coefficients of $t_{\mu}$, we get $([u, v], w)=(u,[v, w])$.

Suppose $\nu=q \mu$, i.e., $\nu=0, \mu,-2 \mu$ or $-\frac{1}{2} \mu$. We first consider the case $\nu \neq 0$. Let $\lambda=\frac{1}{2} \mu$ if $\mu$ is not reduced and $\lambda=\mu$ otherwise. Let $\mathfrak{g}_{1}=\left\langle L_{ \pm \lambda}^{0}\right\rangle$ be the 3 -dimensional split simple Lie algebra of type $A_{1}$ and $\mathfrak{h}_{1}=\left\langle\lambda^{\vee}\right\rangle$ its Cartan 
subalgebra. Let $\Delta_{1}=\{ \pm \lambda, \pm 2 \lambda\}$. We let the subalgebra $M$ of $L$ generated by $L_{ \pm \lambda}^{0}, L_{ \pm \mu}^{ \pm g}, L_{ \pm \nu}^{ \pm k}$ and $L_{ \pm(\mu+\nu)}^{ \pm(g+k)}$. Then

$$
M=\bigoplus_{\xi \in \Delta_{1} \cup\{0\}} \bigoplus_{l \in\langle g, k\rangle} M_{\xi}^{l}
$$

is a Lie ( 1 or 2 )-torus of type $\Delta_{1}$, i.e., type $\mathrm{BC}_{1}$ (or $\mathrm{A}_{1}$ ), with grading pair $\left(\mathfrak{g}_{1}, \mathfrak{h}_{1}\right)$, and $u \in L_{\mu}^{g}=M_{\mu}^{g}, v \in L_{\nu}^{k}=M_{\nu}^{k}$ and $w \in L_{-\mu-\nu}^{-g-k}=M_{-\mu-\nu}^{-g-k}$.

Now, by Lemma $6.5, M$ admits a nonzero graded form $(\cdot, \cdot)^{M}$, and by Lemma 2.1, $\left.(\cdot, \cdot)^{M}\right|_{\mathfrak{g}_{1} \times \mathfrak{g}_{1}}$ is nondegenerate. Note that for any $\xi \in \Delta_{1}, t_{\xi}$ is a scalar multiple of $\lambda^{\vee}$, and so $t_{\xi} \in \mathfrak{h}_{1}$. Since $\kappa\left(t_{\xi}, t_{\xi}\right) \neq 0,\left.\kappa(\cdot, \cdot)\right|_{\mathfrak{h}_{1} \times \mathfrak{h}_{1}}$ is nondegenerate, and so $\left.\kappa(\cdot, \cdot)\right|_{\mathfrak{g}_{1} \times \mathfrak{g}_{1}}$ is nondegenerate. Thus there exists $0 \neq$ $c \in F$ such that $\left.c(\cdot, \cdot)^{M}\right|_{\mathfrak{g}_{1} \times \mathfrak{g}_{1}}=\left.\kappa(\cdot, \cdot)\right|_{\mathfrak{g}_{1} \times \mathfrak{g}_{1}}$. Using this nonzero graded form $c(\cdot, \cdot)^{M}$, we have, by Lemma 2.1 ,

$$
\left[x_{\xi}^{l}, x_{-\xi}^{-l}\right] \equiv c\left(x_{\xi}^{l}, x_{-\xi}^{-l}\right)^{M} t_{\xi}^{M} \bmod Z(M)
$$

for any $x_{\xi}^{l} \in M_{\xi}^{l}$ and $x_{-\xi}^{-l} \in M_{-\xi}^{-l}$, where $Z(M)$ is the centre of $M$, and $t_{\xi}^{M}$ is a unique element in $\mathfrak{h}_{1}$ such that

$$
\kappa\left(t_{\xi}, h\right)=\xi(h)=c\left(t_{\xi}^{M}, h\right)^{M}=\kappa\left(t_{\xi}^{M}, h\right)
$$

for all $h \in \mathfrak{h}_{1}$. Hence, $t_{\xi}^{M}=t_{\xi}$ for all $\xi \in \Delta_{1}$. Thus, by our definition (7.2) of $(\cdot, \cdot)$, we have for all $\xi \in \Delta_{1}$,

$$
\left(x_{\xi}^{l}, x_{-\xi}^{-l}\right) t_{\xi}+z=\left[x_{\xi}^{l}, x_{-\xi}^{-l}\right]=c\left(x_{\xi}^{l}, x_{-\xi}^{-l}\right)^{M} t_{\xi}+z_{M},
$$

where $z \in Z$ and $z_{M} \in Z(M)$. In particular, $z \in M$, and so $z \in Z(M)$. Since $F t_{\xi} \cap Z(M)=0$, we get

$$
\left(x_{\xi}^{l}, x_{-\xi}^{-l}\right)=c\left(x_{\xi}^{l}, x_{-\xi}^{-l}\right)^{M}
$$

for all $x_{\xi}^{l} \in M_{\xi}^{l}$ and $x_{-\xi}^{-l} \in M_{-\xi}^{-l}$ with $\xi \in \Delta_{1}$. But $c(\cdot, \cdot)^{M}$ is invariant, and so $([u, v], w)=c([u, v], w)^{M}=c(u,[v, w])^{M}=(u,[v, w])$.

We now consider the case $\nu=0$, i.e., $v \in L_{0}^{k}$. So

$$
v=\sum_{\eta \in \Delta, k=s+t}\left[v_{\eta}^{s}, v_{-\eta}^{t}\right]
$$

for some $v_{\eta}^{s} \in L_{\eta}^{s}$ and $v_{-\eta}^{t} \in L_{-\eta}^{t}$. Thus it is enough to show that

$$
\left(\left[u,\left[v_{\eta}^{s}, v_{-\eta}^{t}\right]\right], w\right)=\left(u,\left[\left[v_{\eta}^{s}, v_{-\eta}^{t}\right], w\right]\right)
$$


Suppose first that $\eta \neq q \mu$ for $q \in \mathbb{Q}$. Then

$$
\begin{aligned}
\left(\left[u,\left[v_{\eta}^{s}, v_{-\eta}^{t}\right]\right], w\right) & =\left(\left[\left[u, v_{\eta}^{s}\right], v_{-\eta}^{t}\right], w\right)-\left(\left[\left[u, v_{-\eta}^{t}\right], v_{\eta}^{s}\right], w\right) \quad \text { (Jacobi identity) } \\
& =\left(\left[u, v_{\eta}^{s}\right],\left[v_{-\eta}^{t}, w\right]\right)-\left(\left[u, v_{-\eta}^{t}\right],\left[v_{\eta}^{s}, w\right]\right)
\end{aligned}
$$

(If $\mu \pm \eta \in \Delta$, then $\left\{t_{\mu+\eta}, t_{-\eta}\right\}$ and $\left\{t_{\mu-\eta}, t_{\eta}\right\}$ are linearly independent sets and so use the previous case. If $\mu+\eta \notin \Delta$, then $-\mu-\eta \notin \Delta$, and so $\left[u, v_{\eta}^{s}\right]=0=$ $\left[v_{-\eta}^{t}, w\right]$. If $\mu-\eta \notin \Delta$, then $-\mu+\eta \notin \Delta$, and so $\left[u, v_{-\eta}^{t}\right]=0=\left[v_{\eta}^{s}, w\right]$. Hence the equality holds for any case.)

$$
=\left(u,\left[v_{\eta}^{s},\left[v_{-\eta}^{t}, w\right]\right]\right)-\left(u,\left[v_{-\eta}^{t},\left[v_{\eta}^{s}, w\right]\right]\right)
$$

(since $\left\{t_{\mu}, t_{\eta}\right\}$ and $\left\{t_{\mu}, t_{-\eta}\right\}$ are linearly independent sets)

$$
=\left(u,\left[\left[v_{\eta}^{s}, v_{-\eta}^{t}\right], w\right]\right) \quad(\text { Jacobi identity }) .
$$

For the case $\eta=q \mu$, since $\eta \neq 0$, one can define $\lambda, \mathfrak{g}_{1}, \mathfrak{h}_{1}$ and $\Delta_{1}$ by the same way as in the previous case. Namely, let the subalgebra $N$ of $L$ generated by $L_{ \pm \lambda}^{0}, L_{ \pm \mu}^{ \pm g}, L_{ \pm \eta}^{ \pm s}, L_{ \pm \eta}^{ \pm t}$ and $L_{ \pm \mu}^{ \pm(g+s+t)}$. Then

$$
N=\bigoplus_{\xi \in \Delta_{1} \cup\{0\}} \bigoplus_{l \in\langle g, s, t\rangle} N_{\xi}^{l}
$$

is a Lie $(1,2$ or 3$)$-torus of type $\Delta_{1}$, and $u \in L_{\mu}^{g}=N_{\mu}^{g},\left[v_{\eta}^{s}, v_{-\eta}^{t}\right] \in N_{0}^{s+t}$ and $w \in L_{-\mu}^{-g-s-t}=N_{-\mu}^{-g-s-t}$. Thus, by the same reason as in the previous case, there exists a nonzero graded form $(\cdot, \cdot)^{N}$ on $N$ such that

$$
\left(x_{\xi}^{l}, x_{-\xi}^{-l}\right)=\left(x_{\xi}^{l}, x_{-\xi}^{-l}\right)^{N}
$$

for all $x_{\xi}^{l} \in N_{\xi}^{l}$ and $x_{-\xi}^{-l} \in N_{-\xi}^{-l}$ with $\xi \in \Delta_{1}$. Therefore,

$$
\begin{aligned}
\left(\left[u,\left[v_{\eta}^{s}, v_{-\eta}^{t}\right]\right], w\right) & =\left(\left[u,\left[v_{\eta}^{s}, v_{-\eta}^{t}\right]\right], w\right)^{N}=\left(u,\left[\left[v_{\eta}^{s}, v_{-\eta}^{t}\right], w\right]\right)^{N} \\
& =\left(u,\left[\left[v_{\eta}^{s}, v_{-\eta}^{t}\right], w\right]\right) .
\end{aligned}
$$

Thus, by Lemma 4.1(ii), our bilinear form $(\cdot, \cdot)$ on $L^{\prime}$ extends uniquely on $L$, which is a nonzero graded form.

With (3.1) and Lemma 3.2, we have:

Theorem 7.3. The core of any EALA is a Lie torus over $\mathbb{C}$. Conversely, for any Lie torus $L$ over $\mathbb{C}$ with its centre $Z, L / Z$ is isomorphic to the centreless core of some EALA. 
In particular, by Remark 6.2, we also have the following:

Corollary 7.4. Let $(A,-)$ be any structurable torus over $\mathbb{C}$. Then $K(A,-)$ is isomorphic to the centreless core of some EALA of type $B C_{1}$ or type $A_{1}$ if $A=(A,-)$ is a Jordan torus.

Remark 7.5. (i) As in the case of Theorem 5.2, Theorem 7.2 is true for any Lie $G$-torus if any structurable $G$-torus admits a nonzero graded form. (see Remark 5.3).

(ii) The classification of Lie tori over $F$ of type $\mathrm{C}_{l}, \mathrm{~F}_{4}, \mathrm{G}_{2}$ and $\mathrm{BC}_{l}$ is not yet known though the classification of the cores of EALAs is done, up to central extensions, except for both types $\mathrm{BC}_{1}$ and $\mathrm{BC}_{2}$ (see [BGK], [BGKN], [AG], [Y1], [Y2], [Y4], [Y5] and [ABG2]).

\section{$\S 8 . \quad$ The Root Systems of Lie Tori}

Let $L=\oplus_{\mu \in \Delta \cup\{0\}} \oplus_{g \in G} L_{\mu}^{g}$ be a Lie $G$-torus. For each $\mu \in \Delta \cup\{0\}$, let

$$
S_{\mu}:=\left\{g \in G \mid \mathcal{L}_{\mu}^{g} \neq 0\right\} .
$$

Then

$$
R(L):=\left\{S_{\mu}\right\}_{\mu \in \Delta}
$$

is a reduced root system extended by $G$ (see [Y5]). Also, if $G=\mathbb{Z}^{n}$, then the two concepts, a reduced root system extended by $\mathbb{Z}^{n}$ and an extended affine root system (EARS), coincide (see [Y5]). There is a way to know this fact directly using a nondegenerate graded form of a centreless Lie torus. For this purpose we construct a slightly bigger Lie algebra $\tilde{L}$ (than $L$ ) whose root system is an EARS. By the construction, one can directly see that $R(L)$ is an EARS. The method is the complete analogue of the process of adding central elements and derivations to a loop algebra. Also, such a construction was already explained in [AABGP, Ch.III], but to emphasize that this works for over $F$, not only over $\mathbb{C}$, we repeat the argument briefly.

Let $L=\oplus_{\mu \in \Delta \cup\{0\}} \oplus_{\sigma \in \mathbb{Z}^{n}} \quad L_{\mu}^{\sigma}$ be a centreless Lie $n$-torus over $F$ with grading pair $(\mathfrak{g}, \mathfrak{h})$. Then by Theorem 7.1 and Lemma 2.1, there exists a nondegenerate graded form $(\cdot, \cdot)$ on $L$ such that $\left.(\cdot, \cdot)\right|_{\mathfrak{g} \times \mathfrak{g}}$ is the Killing form $\kappa$ of $\mathfrak{g}$. Let Der $L$ be the Lie algebra of all derivations of $L$. Define $d_{i} \in \operatorname{Der} L$ as $d_{i}(x)=k_{i} x$ for $x \in L^{\sigma}$ and $\sigma=\left(k_{1}, \ldots, k_{n}\right) \in \mathbb{Z}^{n}$. Let

$$
D=F d_{1} \oplus \cdots \oplus F d_{n} \subset \operatorname{Der} L \quad \text { and } \quad C=F c_{1} \oplus \cdots \oplus F c_{n}
$$


be two kinds of $n$-dimensional vector spaces. Let $\tilde{L}=L \oplus C \oplus D$, with new bracket $[,]^{\sim}$ defined as follows:

$$
\begin{aligned}
& {[\tilde{L}, C]^{\sim}=0, \quad[D, D]^{\sim}=0, \quad\left[d_{i}, x\right]^{\sim}=d_{i} x \text { for all } x \in L,} \\
& {[x, y]^{\sim}=[x, y]+\sum_{i=1}^{n}\left(d_{i} x, y\right) c_{i} \quad \text { for all } x, y \in L .}
\end{aligned}
$$

Then $\tilde{L}$ is a Lie algebra. Next we extend the form on $\tilde{L}$ as follows:

$$
\begin{aligned}
& (C, C)=(D, D)=(C, L)=(D, L)=0 \quad \text { and } \quad\left(c_{i}, d_{j}\right)=\delta_{i j} \\
& \quad \text { for all } 1 \leq i, j \leq n
\end{aligned}
$$

Then $(\cdot, \cdot)$ on $\tilde{L}$ is a nondegenerate form. We put $H=\mathfrak{h} \oplus C \oplus D$, and identify the dual space $H^{*}=\mathfrak{h}^{*} \oplus C^{*} \oplus D^{*}$. Let $\left\{\delta_{1}, \ldots, \delta_{n}\right\}$ be the dual basis of $\left\{d_{1}, \ldots, d_{n}\right\}$, and identify $\mathbb{Z}^{n} \subset D^{*}$ by $\left(k_{1}, \ldots, k_{n}\right)=\sum_{i=1}^{n} k_{i} \delta_{i}$. Then,

$$
[d, x]^{\sim}=\sigma(d) x \quad \text { for } d \in D, x \in L^{\sigma} \text { and } \sigma \in \mathbb{Z}^{n} .
$$

For $\alpha \in H^{*}$, let

$$
\tilde{L}_{\alpha}=\left\{x \in \tilde{L} \mid[h, x]^{\sim}=\alpha(h) x \text { for all } h \in H\right\} .
$$

Then,

$$
\tilde{L}=\bigoplus_{\alpha \in H^{*}} \tilde{L}_{\alpha}=\bigoplus_{\mu \in \Delta \cup\{0\}} \bigoplus_{\sigma \in \mathbb{Z}^{n}} \tilde{L}_{\mu+\sigma}
$$

with

$$
\tilde{L}_{0}=H \quad \text { and } \quad \tilde{L}_{\mu+\sigma}=L_{\mu}^{\sigma} \quad \text { for }(\mu, \sigma) \neq(0,0)
$$

Let

$$
\tilde{R}:=\left\{\alpha \in H^{*} \mid \tilde{L}_{\alpha} \neq 0\right\}
$$

Then we have a one to one correspondence between the sets

$$
\tilde{R} \approx R(L) \cup\left\{S_{0}\right\} \quad \text { via } \quad \mu+\sigma \leftrightarrow \sigma \in S_{\mu} .
$$

Since $\left.(\cdot, \cdot)\right|_{H \times H}$ is nondegenerate, one can define the induced form $(\cdot, \cdot)$ on $H^{*}$ by the usual way. Identify the prime field of $F$ with $\mathbb{Q}$ (the field of rational numbers). Let

$$
V:=\sum_{\alpha \in \tilde{R}} \mathbb{Q} \alpha
$$

and let $l$ be the rank of $\Delta$. Then: 
Lemma 8.1. $\quad V$ is an $(l+n)$-dimensional vector space over $\mathbb{Q}$ and the restricted form $\left.(\cdot, \cdot)\right|_{V \times V}$ is positive semidefinite over $\mathbb{Q}$ with radical $N:=$ $\oplus_{i=1}^{n} \mathbb{Q} \delta_{i}$.

Proof. By our construction of the form, we have $(N, V)=0$. Let $V^{\prime}:=$ $\sum_{\mu \in \Delta} \mathbb{Q} \mu$. Then $V=V^{\prime} \oplus N$. So we only need to show that $(\cdot, \cdot)^{\prime}:=$ $\left.(\cdot, \cdot)\right|_{V^{\prime} \times V^{\prime}}$ is positive definite over $\mathbb{Q}$. But $(\cdot, \cdot)^{\prime}$ coincides with the induced form on $\mathfrak{h}^{*}$ restricted to $V^{\prime}$ from the Killing form on $\mathfrak{h}$. Thus one can apply the same argument as in the classical case (see e.g. [H, p.40]).

Let

$$
V_{\mathbb{R}}:=\mathbb{R} \otimes_{\mathbb{Q}} V,
$$

where $\mathbb{R}$ is the field of real numbers. Then one can show that $\left(V_{\mathbb{R}}, \tilde{R}\right)$ is an EARS of type $\Delta$ of nullity $n$. In this sense, one may say that

$\tilde{L}$ is an EALA over $F$ constructed from a centreless Lie torus $L$,

and the core of $\tilde{L}$ is the $n$-dimensional central extension $L^{\prime}:=L \oplus C$ of $L$. Note that $L^{\prime}$ is a Lie torus defining ${L^{\prime}}_{0}^{0}=L_{0}^{0} \oplus C(=\mathfrak{h} \oplus C)$, but $\tilde{L}$ is not a Lie torus since $\tilde{L}$ is not perfect.

The roots in the radical or equivalently the roots in $D^{*}$ are called isotropic roots. The isotropic roots are contained in $\mathbb{Z} \delta_{1} \oplus \cdots \oplus \mathbb{Z} \delta_{n}$.

Remark 8.2. (i) EARSs are classified in [AABGP]. Also, one knows that for some EARS $R$ there does not exist a Lie torus $L$ such that $R(\tilde{L})=R$ (see [AG] or [Y3]).

(ii) By the same way as in $[\mathrm{ABGP}]$, using the classification of EARSs of nullity 1 and the Gabber-Kac Theorem (e.g. [MP]), one knows that a Lie 1torus (not necessarily over $\mathbb{C}$ ) is either a (twisted) loop algebra or its universal central extension.

We have constructed $\tilde{L}$ from $L$ by a $2 n$-dimensional extension. We can make the similar construction by just 2-dimensional extension if $F$ is big enough.

Suppose that $F$ contains at least $n$ linearly independent elements over the prime field $\mathbb{Q}$, say $e_{1}, \cdots, e_{n}$. Let $L$ be a centreless Lie $n$-torus over $F$ as above. In the above construction of $\tilde{L}$, we take

$$
d:=\sum_{i=1}^{n} e_{i} d_{i},
$$


where $d_{i}$ is defined as above, $D=F d$ and $C=F c$. Then we can construct the Lie algebra $\tilde{L}=L \oplus C \oplus D, H=\mathfrak{h} \oplus C \oplus D$, and the nondegenerate form by the same way except

$$
[x, y]^{\sim}=[x, y]+(d x, y) c \quad \text { for all } x, y \in L, \quad \text { and } \quad(c, d)=1 .
$$

Let $\{\delta\}$ be the dual basis of $\{d\}$, and identify $\mathbb{Z}^{n} \subset D^{*}$ by

$$
\left(k_{1}, \ldots, k_{n}\right)=\sum_{i=1}^{n} k_{i} e_{i} \delta .
$$

Then the isotropic roots are contained in $\mathbb{Z} e_{1} \delta_{1} \oplus \cdots \oplus \mathbb{Z} e_{n} \delta_{n}$. Hence the dimension of the radical of $V$ defined above is $n$, and so $\left(V_{\mathbb{R}}, \tilde{R}\right)$ is again an EARS of nullity $n$. Similarly, we can construct an EALA whose root system has nullity $n$ by $2 m$-dimensional extension of a Lie $n$-torus for any $1 \leq m<$ $n$. These EALAs in some sense generalize the notion of a degenerate EALA introduced by Gao [G]. In fact, if $F=\mathbb{C}$, we can define $V=\sum_{\alpha \in \tilde{R}} \mathbb{R} \alpha$ instead of $V=\sum_{\alpha \in \tilde{R}} \mathbb{Q} \alpha$ above. Then for $n=2$ and suitable choice of $e_{1}$ and $e_{2}$ above, one can get $\operatorname{dim}_{\mathbb{C}} \sum_{\alpha \in \tilde{R}} \mathbb{C} \alpha=1$, but $\operatorname{dim}_{\mathbb{R}} V=2$. Such an EALA $\tilde{L}$ is called degenerate.

\section{References}

[A] Allison, B. N., A class of nonassociative algebras with involution containing the class of Jordan algebras, Math. Ann., 237 (1978), 133-156.

[Az] Azam, S., Construction of extended affine Lie algebras by the twisting process, Comm. Algebra, 28 (2000), 2753-2781.

[AABGP] Allison, B. N., Azam, S., Berman, S., Gao, Y. and Pianzola, A., Extended Affine Lie Algebras and Their Root Systems, Mem. Amer. Math. Soc., 126 (1997), 603.

[ABG1] Allison, B. N., Benkart, G. and Gao, Y., Lie Algebras Graded by the Root Systems $\mathrm{BC}_{r}, r \geq 2$, Mem. Amer. Math. Soc., 751 (2002), 158.

[ABG2] , Extended affine Lie algebras of type $B C_{l}, l \geq 3$, Preprint.

[ABGP] Allison, B. N., Berman, S., Gao, Y. and Pianzola, A., A characterization of affine Kac-Moody Lie algebras, Comm. Math. Phys., 185 (1997), 671-688

[AH] Allison, B. N. and Hein, W., Isotopes of some nonassociative algebras with involution, J. Algebra, 69 (1981), 120-142.

[AG] Allison, B.N. and Gao, Y., The root system and the core of an extended affine Lie algebra, Selecta Math. (N.S.), 7 (2001), 149-212.

[AY] Allison, B. N. and Yoshii, Y., Structurable tori and extended affine Lie algebras of type $B C_{1}$, J. Pure Appl. Algebra, 184 (2003), 105-138.

[B] Benkart, G., Derivations and invariant forms of Lie algebras graded by finite root systems, Canad. J. Math., 50 (1998), 225-241.

[BGK] Berman, S., Gao, Y. and Krylyuk, Y., Quantum tori and the structure of elliptic quasi-simple Lie algebras, J. Funct. Anal., 135 (1996), 339-389.

[BGKN] Berman, S., Gao, Y., Krylyuk, Y. and Neher, E., The alternative tori and the structure of elliptic quasi-simple Lie algebras of type $\mathrm{A}_{2}$, Trans. Amer. Math. Soc., 347 (1995), 4315-4363. 
[BM] Berman, S. and Moody, R.V., Lie algebras graded by finite root systems and the intersection matrix algebras of Slodowy, Invent. Math., 108 (1992), 323-347.

[BS] Benkart, G. and Smirnov, O., Lie algebras graded by the root system $\mathrm{BC}_{1}, J$. Lie Theory, 13 (2003), 91-132.

[G] Gao, Y., The degeneracy of extended affine Lie algebras, Manuscripta Math., 97 (1998), 233-249.

[H] Humphreys, J.E., Introduction to Lie algebras and representation theory, second edition, Springer-Verlag, New York, 1972.

[K] Kac, V., Infinite dimensional Lie algebras, third edition, Cambridge University Press, 1990.

[M] Mathieu, O., Classification of simple graded Lie algebras of finite growth, Invent. Math., 108 (1992), 455-519.

[MP] Moody, R. V. and Pianzola, A., Lie algebras with triangular decomposition, John Wiley, New York, 1995.

[NY] Neher, E. and Yoshii, Y., Derivations and invariant forms of Jordan and alternative tori, Trans. Amer. Math. Soc., 355 (2002), 1079-1108.

[OZ] Osborn, J. M. and Zhao, K., $\mathbb{Z} \times \mathbb{Z}$-graded Lie algebras containing a Virasoro algebra and a Heisenberg algebra, Comm. Algebra, 29 (2001), 1677-1707.

[S] Schafer, R. D., Invariant forms on central simple structurable algebras, J. Algebra, 122 (1989), 112-117.

[SY] Saito, K. and Yoshii, D., Extended affine root systems IV (Simply-Laced Elliptic Lie Algebras), Publ. RIMS, Kyoto Univ., 36 (2000), 385-421.

[Ya] Yamane, H., A Serre-type Theorem for the Elliptic Lie Algebras with Rank $\geq 2$, Publ. RIMS, Kyoto Univ., 40 (2004), 441-469.

[Y1] Yoshii, Y., Coordinate algebras of extended affine Lie algebras of type $\mathrm{A}_{1}$, J. Algebra, 234 (2000), 128-168.

[Y2] Root-graded Lie algebras with compatible grading, Comm. Algebra, 29 (2001), 3365-3391.

[Y3] Classification of quantum tori with involution, Canad. Math. Bull., 45 (2002), 711-731.

[Y4] Classification of division $\mathbb{Z}^{n}$-graded alternative algebras, J. Algebra, 256 (2002), 28-50.

[Y5] Root systems extended by an abelian group and their Lie algebras, J. Lie Theory, 14 (2004), 371-394. 